\title{
Prevalence of drug resistance and culture-positive rate among microorganisms isolated from patients with ocular infections over a 4-year period
}

This article was published in the following Dove Press journal:

Clinical Ophthalmology

8 April 2013

Number of times this article has been viewed

\author{
Yusuke Shimizu' \\ Hiroshi Toshida' \\ Rio Honda' \\ Asaki Matsui' \\ Toshihiko Ohta' \\ Yousuke Asada ${ }^{2}$ \\ Akira Murakami² \\ 'Department of Ophthalmology, \\ Juntendo University Shizuoka \\ Hospital, Shizuoka, Japan; \\ ${ }^{2}$ Department of Ophthalmology, \\ Juntendo University School of \\ Medicine, Tokyo, Japan
}

Purpose: To investigate the microbial isolates from patients with ocular infections and the trend in the emergence of levofloxacin-resistant strains over the past four years from 2006 to 2009 retrospectively.

Patients and methods: The subjects were 242 patients with ocular infections or traumas treated in our hospital including outpatients, inpatients, and emergency room patients. Most of them needed urgent care presenting with eye complaints, traumas, or decreased vision. Clinical samples were obtained from discharges, corneal, conjunctival tissues or vitreous fluid or aqueous humor, and cultured. Items for assessment included the patient's age, the diagnosis, the prevalence of isolated bacteria, and the results of susceptibility tests for levofloxacin (LVFX) cefamezin (CEZ), gentamicin (GM) and vancomycin. This information was obtained from the patients' medical records.

Results: There were 156 male patients and 86 female patients who were aged from 2 months old to 94 years old and mean age was $56.8 \pm 24.2$ years. Of the 242 patients, $78(32.2 \%)$ had positive cultures. The culture-positive rate was significantly higher in male patients than female in total $(P=0.002)$ and in patients with corneal perforation $(P=0.005)$. Corneal perforation was the highest culture-positive rate $(60.0 \%)$, followed by orbital cellulitis $(56.5 \%)$, blepharitis $(50.0 \%)$, dacryoadenitis $(45.5 \%)$, conjunctivitis $(38.2 \%)$, infectious corneal ulcer $(28.5 \%)$ and endophthalmitis (24.7\%). LVFX-resistant strains accounted for 40 out of a total of 122 strains (32.8\%), and the minimum inhibitory concentration (MIC) was significantly higher in LVFX and GM compared with the other antibiotics. There were no vancomycin-resistant strains.

Conclusion: Attention should be paid to a possible future increase of strains with resistance to LVFX, as commonly prescribed ocular antibiotics bring emergence of resistant bacteria. Although no vancomycin-resistant strains were isolated this drug should be reserved as the last resort, in order to prevent the emergence of vancomycin resistance.

Keywords: ocular infections, drug resistance, levofloxacin, vancomycin, minimum inhibitory concentration

\section{Introduction}

Among the various ocular diseases, certain infections may result in loss of vision, so these are clinically important disorders. ${ }^{1-3}$ In recent years, thanks to the availability of excellent antibiotics and antimicrobial agents, the incidence of infections caused by pathogenic bacteria has declined. In contrast, opportunistic infections caused by bacteria that are usually harmless or of low virulence and infections due to drug-resistant strains have become more prominent over the past 20 to 30 years worldwide. ${ }^{4-6}$ Since the diagnosis and treatment of such infections are complicated, patients may sometimes develop refractory disease. ${ }^{?}$
Correspondence: Hiroshi Toshida Department of Ophthalmology, Juntendo University Shizuoka Hospital, I 29 Nagaoka, Izunokuni, Shizuoka, 410-2295, Japan

Tel +8I 559483 I I I

Fax +8I 55948335 I

Email toshida@juntendo.ac.jp 
In the treatment of ocular infections, it is important to identify (or estimate) the pathogenic bacteria and to provide appropriate antibacterial therapy. It has been reported that ocular infections tend to arise from different causative sources compared with infections at other sites. ${ }^{8}$ The characteristics of clinical isolates from the eyes also tend to be different depending on the location of the pathology, such as the palpebral region, conjunctiva, cornea, lacrimal gland/ lacrimal duct, orbit, or intraocular region. In addition, technologic developments, travel, globalization and migration, as well as changes over time, may influence the trends of these infections. ${ }^{2,3}$ Such trends should be kept in mind when providing treatment for ocular infections.

In order to understand the current status of ocular infections in Japan in the early 21 st century, this study retrospectively investigated the clinical isolates obtained from patients who were diagnosed as having ocular infections, based on data obtained from the medical records. The items investigated and analyzed were the patient's age, the location of the infections and lesions, the bacteria isolated, and the trends of sensitivity to levofloxacin (LVFX), cefamezin (CEZ), gentamicin (GM) and vancomycin. In addition, sensitivity to levofloxacin was compared between each ocular infection.

\section{Materials and methods}

We retrospectively reviewed the medical records of all patients with ocular infections or ocular traumas who were treated at Juntendo University Shizuoka Hospital, Shizuoka, Japan, during the period from January 2006 to December 2009. The total number of patients who underwent culture examination was 242. The subjects included outpatients, inpatients, and emergency room patients. Most of them needed urgent care presenting with eye complaints, traumas and/or decreased vision. In some patients, bacterial culture was done several times by episode of infection or change of the infectious condition.

Samples for culture were collected by ophthalmologists from discharges, corneal or conjunctival tissue, vitreous fluid, or aqueous humor of the eye infections or traumas using a disinfected cotton swab or a scalpel blade under topical anesthesia..$^{9,10}$ In some patients, the examinations were performed from several points simultaneously for example, samples were taken from corneal tissue and discharge in corneal ulcer patients.

The collected materials were routinely stained with Gram stain, and were smeared over the surface of blood, chocolate, Sabouraud's agar, and thioglycolate agar plates (Nissui Pharmaceutical Co, Ltd, Tokyo, Japan) for culture..$^{9-12}$ Cultures were considered positive if colonies grew at the sites of inoculation on the agar plates and bacteria were identified by standard laboratory techniques. Antibiotic sensitivities were determined in accordance with the methods of the National Committee for Clinical Laboratory Standards (NCCLS) using the disc diffusion method ${ }^{13}$ and fully automated microbiology system (RAISUS; Nissui Pharmaceutical Co, Ltd, Tokyo, Japan), and the minimum inhibitory concentration (MIC) was judged to be the lowest concentration of each anti-bacterial agents that substantially inhibited the growth of the organism.

The following data were collected from the medical records of both inpatients and outpatients with ocular diseases and a positive culture; the patient's age, sex, diagnosis, location of the infection, clinical isolates, culture-positive rate for each disease, and sensitivity of each isolate excluding fungi to LVFX, CEZ, GM, and vancomycin. Evaluation of these factors was also done for the following diseases: infectious corneal ulcer, corneal perforation, orbital cellulitis, endophthalmitis, conjunctivitis, dacryoadenitis, and blepharitis.

Statistical analysis was performed using SPSS (SPSS for Windows, version 14.0, SPSS, Chicago, IL, USA). The $2 \times 2$ Chi-square test was employed for comparison of categorical variables. For comparison among multiple groups, the TukeyKramer method was used. Differences were considered significant at $P<0.05$.

\section{Results}

A total number of 242 eyes from 242 patients were diagnosed with ocular infections or traumas at our institution during the study period. The mean age of the patients was $56.8 \pm 24.2$ years, ranging from 2 months to 94 years. There were 156 male patients (64.6\%) and 86 female (35.4\%). When the number of patients with each disease was assessed, the most common disease was infectious corneal ulcer in 103 patients $(42.6 \%)$, followed by endophthalmitis in 52 patients $(21.5 \%)$, conjunctivitis in 39 patients $(16.1 \%)$, dacryoadenitis in 17 patients $(7.0 \%)$, corneal perforation in 12 patients $(5.0 \%)$, orbital cellulitis in 12 patients $(5.0 \%)$, blepharitis in 4 patients $(1.7 \%)$ and others in 3 patients $(1.2 \%)$. In some patients, the culture examination was performed several times.

Microorganisms were isolated from 78 eyes of the 242 eyes. Of 78 eyes, a single isolate was detected in 45 eyes, while there were two isolates in 22 eyes, three isolates in 9 eyes, and four isolates in 2 eyes.

Table 2 shows the number and detection rate of ocular infectious diseases. When males and females were compared, the culture-positive rate was significantly higher in male 
patients $(P=0.002)$. Corneal perforation was the highest culture-positive rate $(60.0 \%)$, followed by orbital cellulitis (56.5\%), blepharitis (50.0\%), dacryoadenitis (45.5\%), conjunctivitis (38.2\%), and infectious corneal ulcer (28.5\%). Endophthalmitis only had a rate of $24.7 \%$ and this was the lowest culture-positive rate. In the patients with corneal perforation, the culture-positive rate was higher for males than for females.

A total of 124 strains were isolated. Among these isolates, 85 strains were gram-positive $(68.5 \%)$ and 37 strains were gramnegative (29.8\%) (Table 2). There were only 2 fungal isolates $(1.6 \%)$ including one strain of Candida albicans. Gram-positive bacteria included methicillin-sensitive coagulase negative Staphylococcus (CNS) (22 strains, 17.7\%), methicillin-resistant CNS (MRCNS) (17 strains, 13.7\%), methicillin-sensitive Staphylococcus aureus (14 strains, 11.3\%), methicillinresistant Staphylococcus aureus (MRSA) (12 strains, 9.7\%), Corynebacterium sp., (9 strains, 7.3\%), Streptococcus $s p$. excluding Streptococcus pneumonia (7 strains, 5.6\%), and Streptococcus pneumoniae (4 strains, 3.2\%). Gramnegative bacteria included were Pseudomonas aeruginosa (12 strains, 9.7\%), Serratia marcescens (7 strains, 5.6\%), Stenotrophomonas maltophilia (3 strains, 2.4\%), Haemophilus influenzae (3 strains, 2.4\%), Klebsiella pneumoniae (3 strains, $2.4 \%$ ), Chryseobacterium indologenes ( 2 strains, $1.6 \%$ ), Proteus mirabilis (2 strains, 1.6\%), Enterococcus faecalis (1 strain, 0.8\%), Delftia acidovorans (1 strain, 0.8\%), Achromobacter $s p$. (1 strain, 0.8\%), Acinetocacter baumannii (1 strain, $0.8 \%$,) and Moraxella sp. (1 strain, 0.8\%).

LVFX-resistant strains accounted for 40 of the 122 bacterial strains $(32.8 \%)$. Table 3 presents the number of strains that were sensitive or resistant to levofloxacin (LVFX) for gram-positive and gram-negative bacteria in each disease category. In the patients with infectious corneal ulcer, 21 gram-positive strains and 24 gram-negative strains were detected. Resistance to LVFX was observed in 3 of the 21 gram-positive strains (14.3\%). In the patients with corneal perforation, all 10 gram-positive strains were CNS including 4 MRCNS and showed LVFX resistance (100.0\%), while all of the gram-negative bacteria $(n=4)$ were sensitive to LVFX. In the patients with orbital cellulitis, 10 gram-positive strains and 3 gram-negative strains were detected, but only 2 grampositive Streptococcus strains showed LVFX resistance (20.0\%). In the patients with endophthalmitis, 15 grampositive strains and 3 gram-negative strains were isolated, and 6 of the 15 gram-positive strains were resistant to LVFX (40.0\%). In the patients with conjunctivitis, 15 gram-positive strains and 5 gram-negative strains were found, with LVFX resistance being shown by 10 strains (66.7\%) and 1 strain (20.0\%), respectively. In the patients with dacryoadenitis, 10 gram-positive strains were isolated and 6 of these strains showed resistance to LVFX (60.0\%).

The MIC of LVFX, GM and CEZ for the clinical isolates was compared between each ocular infectious disease (Table 4). When the MIC of LVFX was compared between each disease, the value for isolates from patients with corneal perforation was significantly higher than that for isolates from the others $(P<0.01)$. Similar result was obtained for GM $(P<0.01)$. In contrast, no significant differences between the groups were found for CEZ. All of the bacterial isolates showed sensitivity to vancomycin and no resistant strains were detected.

\section{Discussion}

One of the objectives of this study was to perform a comprehensive investigation of the bacteria causing ocular infections

Table I The number and detection rate of ocular infectious diseases

\begin{tabular}{|c|c|c|c|c|c|c|c|}
\hline & \multicolumn{2}{|l|}{ Total } & \multicolumn{2}{|l|}{ Male } & \multicolumn{2}{|l|}{ Female } & \multirow{2}{*}{$\begin{array}{l}\text { Differences } \\
\text { between genders } \\
P \text { value }\end{array}$} \\
\hline & Number & $\begin{array}{l}\text { Detection } \\
\text { rate }(\%)\end{array}$ & Number & $\begin{array}{l}\text { Detection } \\
\text { rate }(\%)\end{array}$ & Number & $\begin{array}{l}\text { Detection } \\
\text { rate }(\%)\end{array}$ & \\
\hline Infectious corneal ulcer & $51 / 179$ & $28.5 \%$ & $31 / 91$ & $34.1 \%$ & $20 / 88$ & $22.7 \%$ & $P=0.929$ \\
\hline Corneal perforation & $15 / 25$ & $60.0 \%$ & $15 / 20$ & $75.0 \%$ & $0 / 5$ & $0.0 \%$ & $P=0.005^{* *}$ \\
\hline Orbital cellulitis & $13 / 23$ & $56.5 \%$ & $8 / 12$ & $66.7 \%$ & $5 / 11$ & $45.5 \%$ & $P=0.546$ \\
\hline Endophthalmitis & $20 / 81$ & $24.7 \%$ & $9 / 37$ & $24.3 \%$ & $\mathrm{II} / 44$ & $25.0 \%$ & $P=0.85 I$ \\
\hline Conjunctivitis & $21 / 55$ & $38.2 \%$ & $9 / 18$ & $50.0 \%$ & $12 / 37$ & $32.4 \%$ & $P=0.336$ \\
\hline Dacryoadenitis & $10 / 22$ & $45.5 \%$ & $4 / 6$ & $66.7 \%$ & $6 / 16$ & $37.5 \%$ & $P=0.348$ \\
\hline Blepharitis & $2 / 4$ & $50.0 \%$ & $2 / 3$ & $66.7 \%$ & $0 / 1$ & $0.0 \%$ & $P=1.000$ \\
\hline others & $3 / 31$ & $9.7 \%$ & $3 / 18$ & $16.7 \%$ & $0 / 13$ & $0.0 \%$ & $P=0.925$ \\
\hline Total & $135 / 420$ & $32.1 \%$ & $81 / 205$ & $39.5 \%$ & $54 / 215$ & $25.1 \%$ & $P=0.002^{* * *}$ \\
\hline
\end{tabular}

Notes: The detection rate in this table was calculated by total number of examination. Data were statistically analyzed by $2 \times 2$ Chi square test. Differences between sexes were considered at $P<0.05$; **P $<0.01$, ***P $<0.005$. 


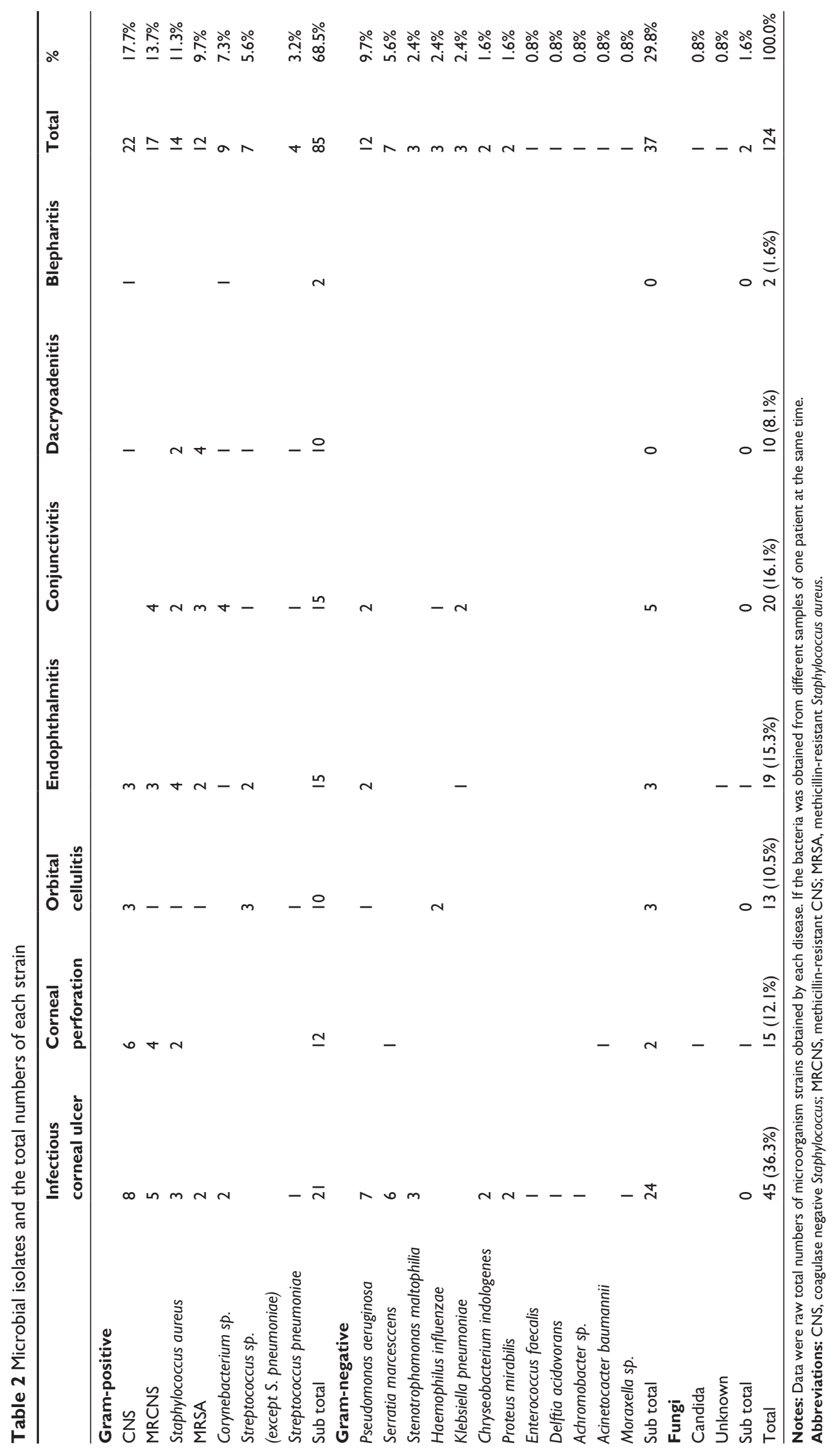




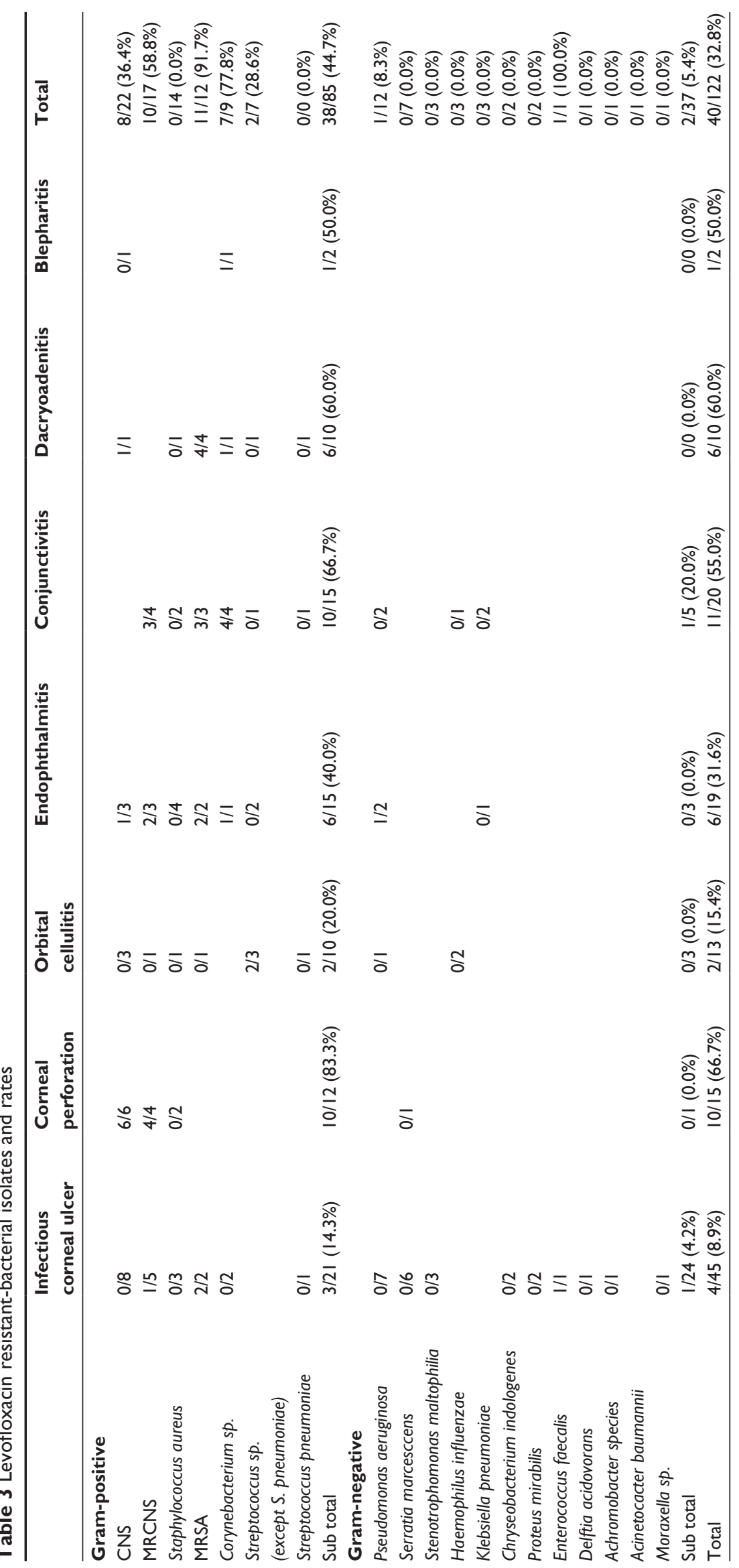


Table 4 The minimum inhibitory concentration

\begin{tabular}{lllr}
\hline & LVFX & GM & \multicolumn{1}{l}{ CEZ } \\
\hline Infectious corneal ulcer & $1.94 \pm 2.16$ & $5.14 \pm 5.66$ & $19.14 \pm 14.93$ \\
Corneal perforation & $5.93 \pm 3.40^{* *}$ & $13.7 \pm 5.45^{* *}$ & $15.67 \pm 14.94$ \\
Orbital cellulitis & $1.86 \pm 2.24$ & $4.33 \pm 5.72$ & $7.00 \pm 12.25$ \\
Endophthalmitis & $2.42 \pm 2.68$ & $5.50 \pm 6.26$ & $11.63 \pm 14.20$ \\
Conjunctivitis & $4.00 \pm 3.14$ & $7.54 \pm 6.98$ & $14.00 \pm 14.83$ \\
Dacryoadenitis & $3.63 \pm 3.62$ & $11.7 \pm 6.74$ & $17.00 \pm 16.43$ \\
\hline
\end{tabular}

Notes: The MIC of LVFX, GM and CEZ for the clinical isolates from each ocular infectious disease. The mean MIC of LVFX and GM for isolates from patients with corneal perforation was significantly higher than that for isolates from the others $(P<0.0$ I, respectively). Data were statistically analyzed by Tukey-Kramer method. Differences were considered significant at $P<0.05 ; * * P<0.01$.

Abbreviations: LVFX, levofloxacin; GM, gentamicin, CEZ, cefamezin; MIC, minimum inhibitory concentration.

as previously reported. ${ }^{1,2}$ Accordingly, in addition to patients with infectious corneal ulcer, corneal perforation, conjunctivitis, dacryoadenitis, and orbital cellulitis, patients who had endophthalmitis were also included. Since the nature of endophthalmitis is essentially different from these other ocular disorders, ${ }^{10}$ it may represent a target for independent investigation. However, in order to understand the overall trends of ocular diseases, we also assessed it in the current study. In general, the incidence of palpebral infections is high, although there were only two patients with blepharitis whose clinical samples were obtained in the present study.

There was a high culture-positive rate in patients with corneal perforation and in patients with orbital cellulitis, with the rate being over $50 \%$. However, the number of clinical samples obtained for these diseases was 25 and 23 , respectively, which was relatively small. On the other hand, the culture-positive rate was low in patients with endophthalmitis, infectious corneal ulcer, and conjunctivitis, but the number of clinical samples was 55. In the patients with endophthalmitis and infectious corneal ulcer, bacterial culture examinations were frequently conducted because bacteria were difficult to isolate and this may have led to a low culture-positive rate. Conjunctivitis is one of the ocular diseases that is most frequently encountered in daily practice, and ophthalmic antibacterial agents are often prescribed. It has been reported that if antibacterial agents have been used, the bacterial isolation rate decreases. ${ }^{6}$ In addition, a history of treatment with antibacterial agents tends to be associated with a higher culture-positive rate of resistant strains, which might also have influenced our results. ${ }^{14,15}$

We focused on investigating the trends of resistance to LVFX because it was one of the most frequently prescribed antibacterial ophthalmic agents for preoperative disinfection. ${ }^{16}$ In the commonly prescribed ocular antibiotics, however, emergence of resistant bacteria is of concern.
The antibacterial activity of new quinolones is generally superior against gram-negative bacteria, but LVFX is claimed to show relatively strong activity against gram-positive bacteria too. In the current study, LVFX resistance was observed in only one strain of Pseudomonas aeruginosa and one strain of Enterococcus faecalis among gram-negative bacteria. Among gram-positive bacteria, however, LVFX resistant strains were seen in all species. This suggests that there is increasing LVFX resistance among gram-positive bacteria. The LVFX resistance rate in the present study $(32.8 \%)$ was higher when comparing to normal conjunctival sac bacterial flora (14.0\%) in our previous study. ${ }^{10}$ Bacteria isolated from the conjunctival sac in healthy person are also thought to be nonpathogenic. ${ }^{17}$ In contrast, antibiotic resistance among ocular isolates was higher in the present study as compared with the previous report. ${ }^{18}$ The result obtained in the present study supports the postulation that usage of antibiotics may contribute to development of drug resistances.

Representative bacteria isolated from patients with infectious corneal ulcer are Staphylococcus aureus, Pneumococcus, Pseudomonas aeruginosa, and Moraxella. ${ }^{19,20}$ In the present study, CNS, Pseudomonas aeruginosa, and Serratia were the top three bacterial species. Although this result is different from previous reports on the trends in Japan, it is similar to the findings of our investigations conducted from 1999 through $2003 .{ }^{9}$ This suggests that the causative bacteria of infectious corneal ulcer might have changed in recent years. The prevalence of resistance to LVFX was relatively low and only three of the 21 gram-positive strains (14.3\%) showed resistance. This may be related to the relatively short duration of treatment with antibacterial ophthalmic agents prior to the onset of infectious corneal ulcer.

In contrast, the number of LVFX-resistant strains was ten of twelve (83.3\%) among gram-positive bacteria in patients with corneal perforation, and this may have been due to the fact that many of these patients received antibacterial agents prior to the occurrence of perforation. Among the patients with corneal infections, differences in the pattern of clinical isolates were small, but the ratio of LVFX resistance was higher. Because of this, in patients who progress to corneal perforation, we should be careful about the emergence of resistant strains. In fact, the mean MIC of LVFX for the isolates from patients with corneal perforation in this study was significantly higher than that for the clinical isolates from infectious corneal ulcer or from orbital cellulitis. Since the data for GM also demonstrated a similar trend, once resistance to a single agent has been acquired, there is a possibility of bacteria acquiring multidrug resistance, even to drugs of 
different classes, as reported previously. ${ }^{21}$ LVFX resistance was observed in only one of the ten strains (10.0\%) from patients with orbital cellulitis. This may have been because antibacterial agents are not frequently administered prior to the onset of this disease.

Although endophthalmitis resulted in poor visual results or eye removal in the past, it is not a refractory disease.

Endophthalmitis was one of the refractory diseases which resulted in poor visual results or eye removal. Recent developments and advances in effective antibiotics and vitreous surgeries, however, have led to the final visual outcome being markedly improved. ${ }^{22}$ In our cases, as treatments vitrectomy with intraocular irrigation with antibiotics and injection procedure with intravitreal vancomycin and ceftazidime was done in all cases as in the previous study, and infections then disappeared. ${ }^{23}$ We did not administer intracameral antibiotics, because it was not common practice in Japan. ${ }^{24}$ In the present study, however, LVFX resistance was found in six of the 15 gram-positive strains $(40.0 \%)$ in the patients with endophthalmitis and the rate was slightly higher than infectious corneal ulcer and orbital cellulitis. It is more likely that strong antibacterial agents will be administered to these patients over a longer period.

It may be no exaggeration to state that conjunctivitis is the most common external ocular infection seen in daily ophthalmologic practice. In patients who show minor eye discharge and hyperemia that is suggestive of infectious conjunctivitis, ophthalmic antibacterial agents are often prescribed without careful consideration and even without performing isolation or culture of bacteria. Because of this casual treatment paradigm, resistant strains are considered to be generated. This assumption is underscored by the finding that LVFX resistance was present in ten of the 15 grampositive strains $(66.7 \%)$ from patients with conjunctivitis, which was high after corneal perforation $(83.3 \%)$ in this study.

Dacryoadenitis frequently occurs in the newborn and in elderly women. ${ }^{25,26}$ In this study, there was 1 female infant ( 2 months old female) and nine of the ten patients were aged 60 years or older. The number of female and male patients was five and four, respectively, and there was no gender difference. Due to the limited number of patients, however, we cannot conclude if this is the latest trend for dacryoadenitis from the findings of the current study. In patients with dacryoadenitis, extrusion of pus from the lacrimal gland and discharge suggest the site of infection, but it tends to be misdiagnosed as conjunctivitis. Accordingly, since antibacterial agents are frequently prescribed, it is considered that the incidence of resistant strains becomes higher. Among gram-positive bacteria, we found LVFX resistance in 6 of the 10 strains $(60.0 \%)$, which was the second highest resistance rate next to conjunctivitis. In previous reports, it has been stated that anaerobic strains are common. However, such bacteria were not isolated in the present study, but this may have been because anaerobic culture was not specified for the clinical samples.

Generally, male and female patients contributed equal percentages to the total isolates. Nonetheless, we could not find the reason why the culture-positive rate in males was higher than in females in the present study. The incidence of resistant strains is expected to continue to increase in the future. With the introduction of new drugs, the bacteria causing each type of infection may change and drug resistance trends will definitely change as well. It is important to understand these trends to successfully manage not only ocular infections but also infection in general. This study provides useful data for understanding the trends of ocular infections in Japan around 2010. Vancomycin is considered to be the treatment of last resort for multi-drug resistant bacteria such as MRSA and MRCNS. Indeed, the present study did not identify any resistance to vancomycin among both gram-negative and gram-positive bacteria isolated from all types of ocular infections and sensitivity to this drug was confirmed. Accordingly, vancomycin can still be positioned as the treatment of last resort even for resistant bacteria. Vancomycin ophthalmic ointment was tested for several years ${ }^{27,28}$ and released in Japan in 2010. Its excessive prescription since then may result in the emergence of vancomycin-resistant strains. ${ }^{29}$ Therefore, any such trend should be carefully monitored. Higher concentration of LVFX $1.5 \%$ began to be distributed in Japan in June, 2011. So, it may also be necessary to pay attention for the emergence of LVFX-resistance carefully.

\section{Disclosure}

The authors declare no conflicts of interest in this work.

\section{References}

1. Snyder RW, Glasser DB. Antibiotic therapy for ocular infection. West J Med. 1994;161(6):579-584.

2. Bertino JS Jr. Impact of antibiotic resistance in the management of ocular infections: the role of current and future antibiotics. Clin Ophthalmol. 2009;3:507-521.

3. Toshida H, Suto C. Ocular bacterial infections. In: Higgins J, Traux D, editors. Eye Infections, Blindness and Myopia. New York: NOVA Science Publishers; 2009:101-118.

4. Keith WW, Heleen HD, Praveen T. Limitations of Current Antibiotics for Ocular Infections. In: Higgins J, Traux D, editors. Eye Infections, Blindness and Myopia. New York: NOVA Science Publishers; 2009:55-67.

5. Harbarth S, Samore MH. Antimicrobial resistance determinants and future control. Emerg Infect Dis. Jun 2005;11(6):794-801. 
6. Croft AC, D'Antoni AV, Terzulli SL. Update on the antibacterial resistance crisis. Med Sci Monit. 2007;13(6):RA103-RA118.

7. Ooishi M, Miyao M. Antibiotic sensitivity of recent clinical isolates from patients with ocular infections. Ophthalmologica. 1997;211(Suppl 1):S15-S24.

8. Evans DJ, McNamara NA, Fleiszig SM. Life at the front: dissecting bacterial-host interactions at the ocular surface. Ocul Surf. 2007;5(3):213-227.

9. Toshida H, Kogure N, Inoue N, Murakami A. Trends in microbial keratitis in Japan. Eye Contact Lens. 2007;33(2):70-73.

10. Suto C, Morinaga M, Yagi T, Tsuji C, Toshida H. Conjunctival sac bacterial flora isolated prior to cataract surgery. Infect Drug Resist. 2012;5:37-41.

11. Inoue N, Toshida H, Mamada N, Kogure N, Murakami A. Contact lensinduced infectious keratitis in Japan. Eye Contact Lens. 2007;33(2): 65-69.

12. Honda R, Toshida H, Suto C, et al. Effect of long-term treatment with eyedrops for glaucoma on conjunctival bacterial flora. Infect Drug Resist. 2011;4:191-196.

13. Clinical and Laboratory Standards Institute. Performance Standards for Antimicrobial Susceptibility Testing. Seventeenth Informational Supplement (M100-S17): Wayne, PA, CLSI, 2007.

14. Miyanaga M, Nejima R, Miyai T, et al. Changes in drug susceptibility and the quinolone-resistance determining region of Staphylococcus epidermidis after administration of fluoroquinolones. $J$ Cataract Refract Surg. 2009;35(11):1970-1978.

15. Kurokawa N, Hayashi K, Konishi M, Yamada M, Noda T, Mashima Y. Increasing ofloxacin resistance of bacterial flora from conjunctival sac of preoperative ophthalmic patients in Japan. Jpn J Ophthalmol. 2002;46(5):586-589.

16. Inoue Y, Usui M, Ohashi Y, Shiota H, Yamazaki T. Preoperative Disinfection Study Group. Preoperative disinfection of the conjunctival sac with antibiotics and iodine compounds: a prospective randomized multicenter study. Jpn J Ophthalmol. 2008;52(3):151-161.

17. McNatt J, Allen SD, Wilson LA, Dowell VR Jr. Anaerobic flora of the normal human conjunctival sac. Arch Ophthalmol. 1978;96(8): $1448-1450$.
18. Fintelmann RE, Hoskins EN, et al. Topical fluoroquinolone use as a risk factor for in vitro fluoroquinolone resistance in ocular cultures. Arch Ophthalmol. 2011;129(4):399-402.

19. Cheung J, Slomovic AR. Microbial etiology and predisposing factors among patients hospitalized for corneal ulceration. Can J Ophthalmol. 1995;30(5):251-255.

20. Varaprasathan G, Miller K, Lietman T, et al. Trends in the etiology of infectious corneal ulcers at the FI Proctor Foundation. Cornea. 2004;23(4):360-364.

21. Bonomo RA, Szabo D. Mechanisms of multidrug resistance in Acinetobacter species and Pseudomonas aeruginosa. Clin Infect Dis. 2006;43(Suppl 2):S49-S56.

22. Bhoomibunchoo C, Ratanapakorn T, Sinawat S, Sanguansak T, Moontawee K, Yospaiboon Y. Infectious endophthalmitis: review of 420 cases. Clin Ophthalmol. 2013;7:247-252.

23. Oshima Y, Kadonosono K, Yamaji H, et al; Japan Microincision Vitrectomy Surgery Study Group. Multicenter survey with a systematic overview of acute-onset endophthalmitis after transconjunctival microincision vitrectomy surgery. Am J Ophthalmol. 2010;150(5): 716-725.

24. Oshika T, Hatano H, Kuwayama Y, et al. Incidence of endophthalmitis after cataract surgery in Japan. Acta Ophthalmol Scand. 2007;85(8): $848-851$.

25. Leone CR Jr. The management of pediatric lacrimal problems. Ophthal Plast Reconstr Surg. 1989;5(1):34-39.

26. Pinar-Sueiro S, Fernández-Hermida RV, Gibelalde A, Martínez-Indart L. Study on the effectiveness of antibiotic prophylaxis in external dacryocystorhinostomy: a review of 697 cases. Ophthal Plast Reconstr Surg. 2010;26(6):467-472.

27. Fukuda M, Hanazome I, Sasaki K. The intraocular dynamics of vancomycin hydrochloride ophthalmic ointment (TN-011) in rabbits. $J$ Infect Chemother. 2003;9(1):93-96.

28. Eguchi H, Shiota H, Oguro S, Kasama T. The inhibitory effect of vancomycin ointment on the manifestation of MRSA keratitis in rabbits. $J$ Infect Chemother. 2009;15(5):279-283.

29. Gupta M, Durand ML, Sobrin L. Vancomycin resistance in ocular infections. Int Ophthalmol Clin. 2011;51(4):167-181.
Clinical Ophthalmology

\section{Publish your work in this journal}

Clinical Ophthalmology is an international, peer-reviewed journal covering all subspecialties within ophthalmology. Key topics include: Optometry; Visual science; Pharmacology and drug therapy in eye diseases; Basic Sciences; Primary and Secondary eye care; Patient Safety and Quality of Care Improvements. This journal is indexed on Submit your manuscript here: http://www.dovepress.com/clinical-ophthalmology-journal

\section{Dovepress}

PubMed Central and CAS, and is the official journal of The Society of Clinical Ophthalmology (SCO). The manuscript management system is completely online and includes a very quick and fair peer-review system, which is all easy to use. Visit http://www.dovepress.com/ testimonials.php to read real quotes from published authors. 